\title{
Conversation autour de l'éthique en pratique : une carrière de communicante sous le signe d'une réflexion et de préoccupations éthiques
}

Juliette Boidot

Antidox Managing Director, Paris Jean-Claude Domenget Maître de conférences - HDR, Université Bourgogne Franche-Comté jean-claude.domenget@univ-fcomte.fr 
1) A l'occasion du colloque du RESIPROC sur l'éthique, vous êtes intervenue lors d'une table ronde sur l'évolution des métiers et des situations de communication dans les organisations, notamment pour pointer la complexité du positionnement des communicants. Pourriez-vous illustrer cette idée-clé de votre analyse?

Tout le monde pense savoir communiquer, il est donc essentiel, selon moi, d'avoir une approche qui se distingue par sa rigueur, sa cohérence et son caractère stratégique. Tout choix, même graphique, doit idéalement pouvoir être justifié et contribuer à l'atteinte des objectifs de l'entreprise. Les supports de communication sont des outils et ils ne sont, comme leur nom l'indique, que des moyens pour atteindre des objectifs opérationnels ou stratégiques. J'ai une vision très ambitieuse de mon métier. Je ne fais pas de la communication pour la communication mais pour participer, grâce à la mise en œuvre du plan de communication, au développement de l'entreprise.

Le positionnement de la fonction communication dans les entreprises est un point extrêmement important. Bien que souvent décrite comme stratégique, cette fonction n'apparaît que trop rarement dans les comités de direction. La direction de la communication est souvent rattachée à une autre fonction support telle que les ressources humaines. Or comment avoir une vision stratégique et la véhiculer si l'on n'est pas impliqué en amont dans l'élaboration de cette stratégie ?

2) Vous avez également évoqué une autre expérience, celle de la gestion d'une communication de crise, à travers le cas très médiatique du Mediator. "Le communicant doit comprendre et travailler sur les enjeux de tous les acteurs». Pouvez-vous préciser votre pensée? Comment se préoccuper d'éthique lorsque les intérêts des différentes parties prenantes sont contradictoires?

Il est effectivement important de comprendre en profondeur les intérêts à agir des différentes parties-prenantes. Le cas du Mediator est à cet effet très intéressant. Lorsque j'ai été recrutée par les laboratoires Servier, l'une des principales missions qui m'avaient été confiées par la direction était de défendre la réputation du groupe, de protéger son image et celle de ses collaborateurs. Les patients, victimes, associations et lanceurs d'alerte souhaitaient que la responsabilité de Servier soit reconnue et que les dommages causés par la prise de Mediator soient indemnisés. Tout en respectant les victimes et en cherchant à les indemniser de la façon la plus juste et la plus rapide possible, les équipes de juristes et d'avocats devaient toujours avoir en tête les potentielles répercussions de leurs actions sur l'issue de procès en cours tant au civil qu'au pénal. L'État, les pouvoirs publics et les organismes de contrôle devaient eux aussi préserver leur image et jouer en toute transparence leur 
rôle de régulateur. Les journalistes, au-delà de l'information factuelle, voulaient des scoops. Les médecins qui avaient prescrit ce médicament, parfois pour de mauvaises raisons, cherchaient à se protéger.

Dans ce contexte, j'ai proposé un positionnement basé sur la responsabilité et la transparence des informations :

- Des faits et des chiffres avec la publication mensuelle du nombre de victimes indemnisées et des montants versés ;

- La reprise des relations avec la presse et en premier lieu l'AFP, à qui nous avons proposé de rencontrer les avocats et juristes hors «crise» pour leur permettre de mieux comprendre tous les aspects de l'affaire ;

- Une information régulière des salariés pour leur permettre d'anticiper les violentes attaques régulièrement véhiculées par les médias.

Un autre exemple intéressant est la gestion par l'AP-HP (Assistance Publique Hôpitaux de Paris) de la communication associée à la gestion de l'accident de train de Brétigny. Quels étaient les enjeux pour chacun des acteurs? La direction de l'AP-HP souhaitait véhiculer une image positive de l'institution. Les journalistes voulaient interviewer médecins et victimes. Les médecins du Samu de Paris (service d'urgence de l'AP-HP) étaient quant à eux occupés à soigner les blessés. Nous avions aussi à cœur de préserver leurs conditions de travail ainsi que l'intimité des victimes et de leurs proches. Il a donc été décidé en accord avec la direction :

- d'organiser, le lendemain de l'accident, un point presse au Samu ;

- de solliciter le directeur du SAMU qui a accepté de répondre aux questions des journalistes.

Grâce à cette initiative, les victimes dans les hôpitaux (dont les portes étaient fermées aux journalistes) ont été préservées, les médecins ont pu faire leur travail, les journalistes ont eu des images qui ont contribué à renforcer la notoriété de l'APHP.

Pour conclure, je dirais que dans les deux cas, nous avons trouvé le moyen de communiquer de façon éthique tout en veillant aux intérêts de chacune des partiesprenantes.

3) Selon vous, le communicant a une déontologie propre et il n'y a aucune incompatibilité entre le fait de nourrir une réflexion et des préoccupations éthiques et le fait de travailler dans la communication. Existe-t-il une éthique du communicant et quelle est-elle?

Je dirais que chacun a ses propres repères déontologiques et doit chercher à les respecter dans son métier. L'image du communicant est malheureusement bien mauvaise. Sur une échelle de crédibilité, la parole du communicant se situe tout en bas. À l'hôpital, un médecin sera toujours plus crédible qu'un administratif, luimême plus crédible qu'un communicant. Or, lorsqu'une crise survient à l'hôpital, c'est souvent au communicant d'accompagner médecins et direction pour éviter la 
langue de bois, quitte parfois à les challenger en les invitant à dire qu'ils ne savent pas répondre à certaines questions. Il s'agit alors d'être fidèle à son éthique personnelle. Pour communiquer efficacement et convaincre l'ensemble de mes publics, il me faut être moi-même convaincue. Par ailleurs, j'ai pu tester tout au long de ma carrière l'efficacité d'une approche fondée sur la responsabilité et la transparence. Selon moi, c'est ainsi que l'on construit sa crédibilité sur le long terme.

4) Cet enjeu de la crédibilité du communicant est au fondement de ce numéro de Communication et professionnalisation. Nous sommes partis du constat d'une défiance $d u$ public envers les communicants, considéré comme des professionnels non crédibles. Pourquoi le métier de communicant souffre-t-il tout particulièrement d'un déficit de confiance?

La communication est un métier encore mal défini qui a longtemps été associée à l'événementiel et aux petits fours. Dans ces conditions, comment le communicant peut-il redonner de la crédibilité à sa fonction? Il doit tout d'abord défendre une approche très rigoureuse de la communication, notamment lorsqu'il travaille dans des environnements scientifiques et techniques, avec des chercheurs, des ingénieurs ou des médecins. La communication doit être au service d'un objectif stratégique et favoriser le développement de l'entreprise. Ainsi, pour promouvoir l'image d'un groupe industriel, de ses produits et services, il est primordial de commencer par définir l'ADN de l'organisation, les cultures qui la définissent et l'irriguent. L'ADN d'une organisation doit correspondre à une réalité reconnue aussi bien en interne par les collaborateurs qu'en externe par les clients.

Prenons un exemple dans le domaine de l'électronique: il s'agissait de faire connaître et promouvoir de nouveaux connecteurs utilisés dans l'équipement des véhicules électriques. Après de longs échanges avec des représentants de différents corps de métiers de l'entreprise, mon équipe et moi avons proposé une campagne de presse qui se concentrait sur la technologie, sur les équipes de R\&D de l'entreprise, sur notre capacité de production, et enfin sur le produit, le connecteur lui-même. Cette campagne a suscité un tel intérêt de la part des clients que les commandes ont dépassé les capacités de production... La direction de la communication a ainsi prouvé son utilité !

5) Les nombreux codes, chartes, accords régulant la profession peuvent-ils changer l'image des communicants? Vous ont-ils été utiles dans votre activité ? Ont-ils pu jouer un rôle de boussole, de garde-fou? 
Selon moi, la solution ne tient pas dans des codes, des chartes déontologiques propres aux communicants. Les règlements intérieurs des entreprises doivent intégrer un véritable code éthique et s'appliquer à tout le monde sans distinction. Je plaide en faveur d'un code of business conduct unique, signé tous les mois par les managers qui s'engagent ainsi à ne pas enfreindre certaines règles et à signaler tout manquement. Un tel code est un vrai guide et un garde-fou utile. Il m'est arrivé qu'on me demande d'agir dans un sens qui était pour moi contraire à l'intérêt de la société. Fallait-il abonder? Fallait-il mentir? Ce sont des questions que tous les salariés sont amenés à se poser. Et le communicant n'est pas différent des salariés de ce point de vue, si ce n'est que sa fonction est plus visible et qu'il est plus exposé par définition. De fait, l'utilité d'un code déontologique spécifique à la fonction « communication » ne m’apparaît pas comme une évidence.

6) Concernant la régulation de la profession, Ritha Cossette indique que la reconnaissance du professionnalisme des communicants repose en partie sur l'adoption de normes éthiques. Identifiez-vous derrière ce plaidoyer pour une régulation accrue un risque de limitation de la liberté d'invention, de création, d'innovation, telle que le déplorait Jean Devèze?

C'est un risque auquel je n'ai jamais été confrontée. J'insiste toujours sur la rigueur, mais je n'oublie pas la créativité. De toute évidence, elle est essentielle. C'est en elle que réside l'attrait des métiers de la communication. C'est grâce à celle-ci qu'un bon communicant trouvera des solutions pragmatiques pour résoudre des problèmes complexes. La communication est affaire d'innovation, elle doit attirer l'attention et donc surprendre, se renouveler. Dans un groupe international, où la barrière des langues est un réel frein à la communication, pourquoi ne pas inventer un mode de communication purement visuel ? Lorsque l'on souhaite briser la glace entre les équipes de direction des différentes régions ou divisions, quoi de mieux qu'un petit tour dans les bains chauds de Budapest?

7) Les chercheurs relient les questions de stratégie de présence en ligne et d'eréputation à une myriade de notions, telles que la notoriété, la popularité, la crédibilité, l'influence, l'autorité, etc. Quels enjeux éthiques sont liés à ces notions et logiques d'action?

Il me semble que l'e-réputation doit correspondre à une réalité. Mentir sur Internet pour améliorer sa réputation est risqué. La communication des organisations sur les médias sociaux doit participer à la communication globale, et ce de façon cohérente. Il est généralement intéressant de se positionner en tant qu'expert dans son secteur d'activité, de communiquer sur ses produits, mais aussi sur ses métiers. 
Chez Areva, nous avions créé un journal externe «Alternatives : parler autrement de l'énergie » qui serait typiquement un blog aujourd'hui. Le comité de rédaction était composé d'experts indépendants. Toutes les formes d'énergie y étaient présentées, et la parole était donnée tant aux partisans qu'aux détracteurs de chacune d'entre-elles. Ce journal, diffusé à des journalistes et des leaders d'opinion, a permis à Areva d'asseoir sa légitimité en tant qu'acteur crédible sur les grands enjeux énergétiques.

À l'AP-HP, l'actualité nous imposait une communication souvent plus réactive que proactive. Qu'il s'agisse d'une crise sanitaire ou de tout autre sujet lié à la santé, nous étions en permanence sollicités en tant qu'acteur-clé et vivier d'experts. Afin de valoriser tant les personnels que les équipements des hôpitaux ou les progrès médicaux, nous avions créé un webzine qui était relayé via un fil Twitter.

Enfin, j'aborderai la question du droit de réponse. Avec les réseaux sociaux, la parole est débridée et des messages très négatifs sur l'entreprise peuvent circuler. Faut-il se précipiter pour répondre aux polémiques nées sur les médias sociaux sur ces mêmes médias ? Je ne le crois pas. Il est plus efficace de répondre de façon posée et donc asynchrone, en prenant par exemple la parole via un autre média.

8) L'éthique est parfois considérée comme une dimension essentielle de la professionnalisation des communicants, une dimension qui leur permettrait de sortir d'une position de simple exécutant pour jouer un rôle stratégique. Éthique et revalorisation de la fonction communication sont-elles, selon vous, liées?

Dans la communication, et c'est une spécificité de cette profession, on est très rapidement amené à travailler avec les équipes dirigeantes des sociétés. Dès ma première année comme communicante, j'ai travaillé avec les membres du comité de direction notamment pour préparer les discours du PDG ou le rapport annuel. Audelà de l'éthique, il est essentiel d'insister sur la rigueur, qui est primordiale dans ce métier pour asseoir sa crédibilité. Ensuite, pour être crédible, la communication doit sortir de la communication! Elle doit permettre de faire ressortir la vraie nature et les vraies causes des problèmes, aussi bien en situation de crise que dans la routine quotidienne et les périodes de changement. Elle peut contribuer à cela grâce à un véritable travail d'animation, de tissages de liens entre équipes, entre métiers et entre fonctions d'une part, et un travail de formalisation rigoureux d'autre part. De ce point de vue, le rapport annuel est un bon exercice car il permet au communicant de rencontrer beaucoup de parties prenantes et de salariés. Grâce à ces rencontres, il ou elle - peut présenter fidèlement toutes les activités d'une organisation et faire ressortir tout ce qui pourra favoriser la stratégie globale de l'entreprise.

Je souhaite ensuite évoquer la question de la transformation de l'entreprise et des freins au changement. La valeur ajoutée de la communication sur ce sujet est de 
réussir à prendre en compte l'intérêt de tout le monde. Contrairement à une idée reçue, la communication d'une entreprise ne peut être réduite à la voix du directeur, dans une logique top-down. Elle doit aider à la mise en place d'une stratégie globale, qui doit inclure tous les acteurs. Ceci suppose d'aller voir tout le monde, d'écouter toutes les parties concernées, d'échanger, de recueillir des propositions pour l'amélioration des services par exemple.

La communication d'une organisation doit être représentative de l'ensemble de ses composantes tant internes qu'externes et ainsi favoriser le travail au service d'une vision et d'objectifs partagés. À l'hôpital par exemple, il y a grosso modo trois types de populations médicales : les médecins, les personnels soignants, et les cadres hospitaliers qui effectuent des missions de coordination. Or ces derniers comprennent souvent mal leur rôle et ne se sentent donc pas suffisamment légitimes. À l'AP-HP, les réunions managériales se déroulaient entre cadres hospitaliers uniquement. J'aurais souhaité que ces réunions incluent également les médecins et les personnels soignants, pour une meilleure compréhension des rôles des uns et des autres, et une meilleure coordination. Travailler de manière insuffisamment transversale, travailler en silo est pour moi clairement un frein à une évolution constructive des organisations.

9) Les modèles de prise de décision éthique des communicants s'inscrivent dans un contexte organisationnel marqué par la complexité : le communicant doitil légitimer l'action de l'entreprise ou bien doit-il tenter d'organiser et de diffuser une information vraie et sincère?

En France, la culture est plus pyramidale qu'il n'y paraît. Pour traiter les vrais problèmes et les vrais sujets, un bon communicant doit pourtant être capable de challenger tous les porte-paroles de son organisation. J'insiste sur le mot « tous». Ceci est particulièrement vrai en cas de communication de crise, quand il est difficile d'obtenir des réponses précises et sincères. Dans ces cas, il faut oser dire ce qui ne va pas ou bien oser dire qu'on ne sait pas. C'est aussi un problème culturel. Prenons le contre-exemple de la crise autour du nucléaire et de Fukushima au Japon. Dans la culture japonaise, on ne peut pas ne pas savoir. C'est une culture dans laquelle on ne parle pas des erreurs techniques.

\section{0) Enfin, y a-t-il une actualité de la question éthique en communication?}

La question des rapports entre les médias et les communicants est essentielle pour comprendre celle de la décrédibilisation des communicants. Ces rapports sont souvent faits de défiance, d'autant que les journalistes sont également perçus comme de moins en moins crédibles. En outre, on a tendance à assimiler les communicants d'entreprise à des journalistes internes, ce qui embrouille inutilement les choses. 
Enfin, avec la montée en puissance des réseaux sociaux, on peut avoir l'impression que n'importe qui peut s'improviser communicant. Les fakes news constituent un challenge de taille pour nos entreprises et pour nos sociétés. Communiquer, ce n'est pas écrire n'importe quoi sur n'importe quel sujet. Comment trouver de l'information certifiée ? Comment reconnaître le journaliste intègre ? Le système blockchain appliqué aux médias sociaux est une solution qu'il faut étudier: il permettrait que toute information passe par un système de filtres pour être certifiée. Tout le monde contrôlerait ainsi tout le monde, sans qu'il y ait alors besoin de mécanismes ou d'instances supérieures de régulation. Il faut aussi et toujours former les jeunes aux bonnes vieilles méthodes comme le doute cartésien, développer leur esprit scientifique, leur apprendre à questionner le statut de leurs sources d'information. Il faut aussi bien sûr former les jeunes sur l'éthique, les aider à acquérir la culture de leur futur métier de communicant. La sensibilisation aux enjeux éthiques s'acquiert, selon moi, avant tout grâce à l'expérience. D'où l'intérêt pour les étudiants d'écouter et d'échanger avec des professionnels qui viennent leur présenter leur métier et des cas pratiques. J'essaie modestement d'y contribuer quand je réponds aux invitations de responsables de formation ou à des demandes d'interviews d'étudiants ou... de chercheurs. 